\title{
The Interaction between Pesticide Use and Genetic Variants Involved in Lipid Metabolism on Prostate Cancer Risk
}

\author{
Gabriella Andreotti, ${ }^{1}$ Stella Koutros, ${ }^{1}$ Sonja I. Berndt, ${ }^{1}$ \\ Kathryn Hughes Barry, ${ }^{1}$ Lifang Hou, ${ }^{2}$ Jane A. Hoppin, ${ }^{3}$ Dale P. Sandler, ${ }^{3}$ \\ Jay H. Lubin, ${ }^{1}$ Laurie A. Burdette, ${ }^{4}$ Jeffrey Yuenger, ${ }^{4}$ Meredith Yeager, ${ }^{4}$ \\ Laura E. Beane Freeman, ${ }^{1}$ and Michael C. R. Alavanja ${ }^{1}$ \\ ${ }^{1}$ Division of Cancer Epidemiology and Genetics, National Cancer Institute, National Institutes of Health, Rockville, \\ MD 20892-7240, USA \\ ${ }^{2}$ Department of Preventive Medicine and Robert H. Lurie Comprehensive Cancer Center, Feinberg School of Medicine, \\ Northwestern University, Chicago, IL 60208, USA \\ ${ }^{3}$ Epidemiology Branch, National Institute of Environmental Health Sciences, National Institutes of Health, \\ Research Triangle Park, NC 27709, USA \\ ${ }^{4}$ Core Genotyping Facility, NCI-Frederick, SAIC-Frederick Inc., Frederick, MD 21702, USA
}

Correspondence should be addressed to Gabriella Andreotti, andreotg@mail.nih.gov

Received 29 December 2011; Accepted 2 April 2012

Academic Editor: Lars Holmberg

Copyright ( $\odot 2012$ Gabriella Andreotti et al. This is an open access article distributed under the Creative Commons Attribution License, which permits unrestricted use, distribution, and reproduction in any medium, provided the original work is properly cited.

\begin{abstract}
Background. Lipid metabolism processes have been implicated in prostate carcinogenesis. Since several pesticides are lipophilic or are metabolized via lipid-related mechanisms, they may interact with variants of genes in the lipid metabolism pathway. Methods. In a nested case-control study of 776 cases and 1444 controls from the Agricultural Health Study (AHS), a prospective cohort study of pesticide applicators, we examined the interactions between 39 pesticides (none, low, and high exposure) and 220 single nucleotide polymorphisms (SNPs) in 59 genes. The false discovery rate (FDR) was used to account for multiple comparisons. Results. We found 17 interactions that displayed a significant monotonic increase in prostate cancer risk with pesticide exposure in one genotype and no significant association in the other genotype. The most noteworthy association was for ALOXE3 rs3027208 and terbufos, such that men carrying the $\mathrm{T}$ allele who were low users had an OR of $1.86(95 \% \mathrm{CI}=1.16-2.99)$ and high users an OR of $2.00(95 \% \mathrm{CI}=1.28-3.15)$ compared to those with no use of terbufos, while men carrying the CC genotype did not exhibit a significant association. Conclusion. Genetic variation in lipid metabolism genes may modify pesticide associations with prostate cancer; however our results require replication.
\end{abstract}

\section{Background}

Previous studies of prostate cancer have shown elevated rates in agricultural and pesticide manufacturing populations [1, 2]. In the Agricultural Health Study (AHS), a significant excess of prostate cancer was observed among private and commercial pesticide applicators compared to the general population $[3,4]$. Also, use of pesticides, such as phorate [5], fonofos [6], butylate [7], and coumaphos [8], has been linked with an increased risk of prostate cancer among AHS participants with a family history of prostate cancer. We conducted a prostate cancer nested case-control study within the
AHS to examine interactions between prespecified genetic pathways and pesticide exposure. Recent findings from this study have identified significant pesticide interactions for several genetic variants in the 8q24 region [9], xenobiotic metabolism pathway [10], and DNA repair pathways [11, 12]. These studies help elucidate exposure-effect associations by identifying potentially susceptible subgroups. This allows us to better understand potential carcinogenic hazards and furthers public health research on the human health effects of pesticides.

In this analysis, we evaluated single nucleotide polymorphisms (SNPs) in genes related to lipid metabolism since 
there is evidence that lipogenic enzymes and serum lipids may play a role in prostate carcinogenesis via inflammatory, oxidative stress and insulin-resistance mechanisms $[13,14]$. Cellular and molecular studies have reported an overexpression of lipogenic enzymes, such as fatty acid synthase and acetyl-CoA carboxylase, and increased lipogenesis in prostate cancer cells $[15,16]$. Genetic variants involved in fatty acid or lipid metabolism, which have been linked to altered expression of lipogenic enzymes and serum lipid levels [16-18], have also been associated with an excess risk of prostate cancer [19-21]. Furthermore, several pesticides are lipophilic or are metabolized via a lipid-related mechanism $[22,23]$, suggesting that variants of genes involved in lipid metabolism may interact with certain pesticides to increase the risk of prostate cancer. We examined potential geneenvironment interactions between SNPs in genes involved in lipid metabolism and pesticide exposure in a nested casecontrol study within the AHS.

\section{Methods}

2.1. Study Subjects. A detailed description of the AHS cohort has been published [24]. Briefly, 57, 310, or 82\%, of pesticide applicators seeking pesticide licensing in Iowa and North Carolina were enrolled in the study between December 13, 1993 and December 31, 1997. Participants were licensed private pesticide applicators (mostly farmers) residing in Iowa and North Carolina and commercial applicators residing in Iowa. To be eligible for this nested case-control study, participants had to provide a mouth-wash rinse buccal cell sample, and could not have had a previous cancer diagnosis except for non-melanoma skin cancer. Cancer diagnoses were identified by linkage with population-based state cancer registries. Cases were males diagnosed with incident prostate cancer between 1993 and 2004, and controls were males alive at the time of case diagnosis with no cancer diagnosis, except nonmelanoma skin cancer at the time of selection. Eligible controls were randomly selected and frequency matched $2: 1$ to cases by date of birth ( \pm 1 year). All participants were self-identified as Caucasian and confirmed to be of European ancestry using population substructure evaluation and principal component analysis as previously described [9].

2.2. Pesticide Use. Information on pesticide use was obtained from two self-administered questionnaires completed at enrollment (http://aghealth.nci.nih.gov/questionnaires.html). These questionnaires collected comprehensive data on lifetime use of 50 pesticides. Participants were asked how many years ( 1 year or less, $2-5,6-10,11-20,21-30$, or more than 30 years) and how many days (less than $5,5-9,10-19,20$ $39,40-59,60-150$, or more than 150 days) in an average year they applied each chemical. Pesticide use was quantified as ever/never use, lifetime exposure days (years of use $x$ days per year), and intensity weighted lifetime exposure days (lifetime exposure days $\times$ intensity score). The intensity score was computed from an algorithm that took into account exposure-modifying factors of how pesticides were used and applied [25].
2.3. SNP Selection and Genotyping. Germline DNA was extracted from buccal cells using Autopure (QIAGEN) at Sera Care, Frederick, MD. Genotyping was conducted with a custom Infinium BeadChip Assays (iSelect) from Illumina Inc., at the NCI Core Genotyping Facility (http://cgf.nci .nih.gov/operations/multiplex-genotyping.html). Common SNPs (minor allele frequency $\geq 5 \%$ ) with an $r^{2}$ threshold $>0.80$ were selected from the HapMap Project population using the Tagzilla software package that implements a modified tag SNP selection method described by Carlson et al. [26]. The Illumina chip included 26, 512 SNPs, of which 1,858 tag SNPs in 85 genes were related to the lipid metabolism pathway. Blinded duplicate samples (2\%) were genotyped; the concordance of these samples ranged from 96$100 \%$. The overall genotyping rate for lipid metabolism SNPs was between $97 \%$ and $100 \%$. Of the samples genotyped, 108 were removed due to insufficient or poor DNA quality ( 14 cases, 6 controls) or $<90 \%$ completion rate (47 cases, 41 controls), leaving 2,220 (776 cases, 1,444 controls) for statistical analyses.

2.4. Statistical Analysis. We compared the distributions of selected characteristics (age $(<40,40-49,50-59,60-69$, $\geq 70$ ), state of residence (Iowa, North Carolina), applicator type (private, commercial), and first-degree family history of prostate cancer (no, yes)) between cases and controls using a chi-square test.

Associations between the 1,895 lipid metabolism SNPs in 85 genes and prostate cancer risk were assessed using PLINK software [27] using a log-additive genetic model to calculate odd ratios (OR) and $95 \%$ confidence intervals (CI). Variants with a Hardy Weinberg equilibrium $P$ value $>0.001$, a minor allele frequency $\geq 0.1$, and a $P$ trend for the additive effect of each allele $<0.2$ were carried forward for interaction analyses, which totaled 220 SNPs in 59 genes. Pairwise linkage disequilibrium (e.g., $D^{\prime}$ and $r^{2}$ ) between SNPs was evaluated using Haploview 4.2 [28].

The statistical interactions between pesticide use and genetic variants were assessed using the likelihood ratio test in unconditional logistic regression models adjusting for age and state. We examined 39 pesticides that were ever used by at least $10 \%$ of the study population. Exposure for each pesticide was categorized as nonexposed, low, and high exposure, with low- and high-exposure categories based on the median intensity-weighted lifetime days. Genetic variants were coded using a dominant genetic model due to the potential for small sample sizes of variant alleles. We calculated ORs and 95\% CIs for prostate cancer risk and pesticide exposure stratified by genotype (homozygous wild-type, heterozygous and homozygous variant) using unconditional logistic regression models adjusting for age and state. In essence, we adopted a two-stage interaction approach [29], where we first set a cut point on the SNPs we included in the interaction analyses, and then cut point on the $P$ interaction value to increase statistical power.

We used the false discovery rate (FDR) [30] to account for multiple comparisons for the main effect associations and the interactions. For the main effect associations, FDR was calculated considering all SNPs within each gene. 
For the interaction analyses, we calculated FDR in two ways: (1) accounting for the 220 SNPs we evaluated per pesticide, which is the most stringent approach, and (2) accounting for the number of SNPs within each gene per pesticide. Since our a priori hypothesis was an increased risk of cancer with exposure, we focused on statistically significant monotonically increasing ORs with increasing pesticide exposure in one genotype and no significant association in the other genotype (quantitative interaction), versus an interaction with increasing pesticide exposure in one genotype and a decreasing pesticide exposure in the other genotype (qualitative interaction), since the former are considered to be more biologically plausible and less due to chance [31]. Unless otherwise indicated, analyses were conducted using SAS, version 9.1 (SAS Institute, Cary, NC) and AHS data release version P1REL0712.04.

\section{Results}

Characteristics of the study population (see Supplementary Table 1 in Supplementary Material available online at doi:10.1155/2012/358076) and associations between intensity-weighted lifetime days of pesticide use and prostate cancer (Supplementary Table 2) were previously published [9-12]. Briefly, cases and controls were similar in terms of age, state of residence and applicator type, but cases were more apt to have a family history of prostate cancer.

Of the 1,895 lipid metabolism SNPs in 85 genes we examined, 20 SNPs in 8 genes (ABCG5, ALOXE3, AMACR, FOXA3, LRP2, PPARA, TBXAS1, UCP3) were significant using a gene-based FDR $<0.05$ (Table 1). The two most significant SNP-cancer associations were for two highly correlated variants in $A B C G 5, \mathrm{rs} 1835815$, and $\mathrm{rs} 2278356\left(r^{2}=\right.$ 0.89 , FDR $P$ main effect $=0.01$ ).

Among the 220 SNPs in 59 genes and 39 pesticides examined in interaction analyses, we found 16 interactions significant at FDR $P$ interaction $<0.2$ accounting for all 220 SNPs per pesticide. All of these interactions had a significant association between pesticide use and prostate cancer in at least one genotype group $(P$ trend $<0.05), 9$ also had an increased risk of prostate cancer (OR trend > 1.0); and three were monotonic associations (i.e., increasing cancer risk with increasing pesticide use) and quantitative interactions (Table 2). These associations involved one pesticide, terbufos, and three SNPs in three genes (ADIPOR1, ALOXE3, SEC14L2).

Using a less stringent method for accounting for multiple comparisons, we found 139 interactions significant using a gene-based FDR for each of the 39 pesticides examined (FDR $P$ interaction $<0.05)$. Of these, 116 had significant associations between pesticide use and prostate cancer in at least one genotype group $(P$ trend $<0.05)$; 31 resulted in increased prostate cancer risk (OR trend $>1.0$ ) (Supplemental Table 4 ), and 17 were monotonic associations and quantitative interactions (Table 3). These 17 associations involved seven pesticides (atrazine, carbofuran, EPTC, fonofos, glyphosate, petroleum oil/distillate, and terbufos) and 15 SNPs in 11 genes (ABCG8, ACSL5, ADIPOR1, ALOX5, ALOX5AP, ALOX15, ALOXE3, A4GALT, LDLR, LRP1, SEC14L2). The findings from this method are inclusive of those using the more stringent method.

We observed significant interactions with three insecticides, terbufos, fonofos, and carbofuran. Overall, the most noteworthy association (i.e., smallest FDR $P$-interaction and pesticide $P$-trend) was for terbufos and ALOXE3 rs3027208 (Table 3). Men carrying the variant $\mathrm{T}$ allele who were low users of terbufos had an OR of $1.86(95 \% \mathrm{CI}=1.16-2.99)$ and high users an OR of $2.00(95 \% \mathrm{CI}=1.28-3.15)$ compared to no use of terbufos $(P$ trend $=0.001)$, while men carrying the CC genotype did not exhibit a significant association (FDR $P$ interaction $=0.01$ ). This association persisted after adjusting for ALOXE3 rs6503086, which had a significant main effect association with prostate cancer (FDR $P$ trend $=0.02)$ but was not correlated with $\mathrm{rs} 3027208\left(r^{2}=0.02\right)$. Terbufos also interacted significantly with SEC14L2 rs2072159 (FDR $P$ interaction $=0.01$ ) and ADIPOR1 rs12733285 (FDR $P$-interaction $=0.02$ ). Fonofos interacted significantly with ABCG8 rs4953028, such that men carrying the GG genotype who were low users of fonofos had an OR of $1.73(95 \%$ $\mathrm{CI}=0.99-3.00)$ and high users an OR of $1.94(95 \% \mathrm{CI}=$ 1.17-3.20) compared to no use of fonofos ( $P$ trend $=$ 0.004), while men carrying the variant A allele did not exhibit a significant association $($ FDR $P$ interaction $=0.02)$. Carbofuran interacted significantly with A4GALT rs8136914 $($ FDR $P$ interaction $=0.03)$ and with $L D L R$ rs8110695 (FDR $P$ interaction $=0.04$ ).

We observed significant interactions with four herbicides, EPTC, petroleum oil/distillates, glyphosate, and atrazine. Among these, the most noteworthy association (i.e., smallest FDR $P$ interaction and pesticide $P$ trend) was for EPTC and ALOX15 rs916055. Men carrying the G allele who were low users of EPTC had an OR of 1.47 (95\% CI $=0.98-$ $2.22)$ and high users an OR of $1.63(95 \% \mathrm{CI}=1.06-2.50)$ compared to no use of EPTC ( $P$ trend $=0.01)$, while men carrying the A allele did not exhibit a significant association $($ FDR $P$ interaction $=0.01)$. Petroleum oil interacted with variants in three genes (ALOX5, LRP1, and ACSL5). The most significant interaction for petroleum oil was with ALOX5 rs7099684, such that men carrying the TT genotype who were low users of petroleum oil had an OR of $1.19(95 \% \mathrm{CI}=$ $0.76-1.86)$ and high users an OR of $1.56(95 \%$ CI $=1.03-$ 2.38) compared to no use of petroleum oil $(P$ trend $=0.03)$, while men carrying the A allele did not exhibit a significant association (FDR $P$-interaction $=0.02$ ). Petroleum oil interacted with four LRP1 SNPs (rs1800159, rs6581128, rs795957, rs7978567) that are moderately to highly correlated $\left(r^{2}=\right.$ 0.56-0.88). The most significant association (i.e., smallest FDR $P$-interaction and $P$-trend) was for LRP1 rs1800159, such that men carrying the A allele who were low users of petroleum oil had an OR of $1.26(95 \% \mathrm{CI}=0.80-2.00)$ and high users an OR of $1.76(95 \% \mathrm{CI}=1.12-2.77)$ compared to no use of petroleum oil ( $P$ trend $=0.01$ ), while men carrying the $\mathrm{G}$ allele did not exhibit a significant association (FDR $P$ interaction $=0.03$ ). Petroleum oil also interacted significantly with two ACSL5 SNPs (rs876873, rs120985760) that are highly correlated $\left(r^{2}=0.89\right)$. Other noteworthy associations included the interactions between glyphosate and ALOX5AP rs9579645 (FDR $P$ interaction $=0.02)$, and between atrazine 


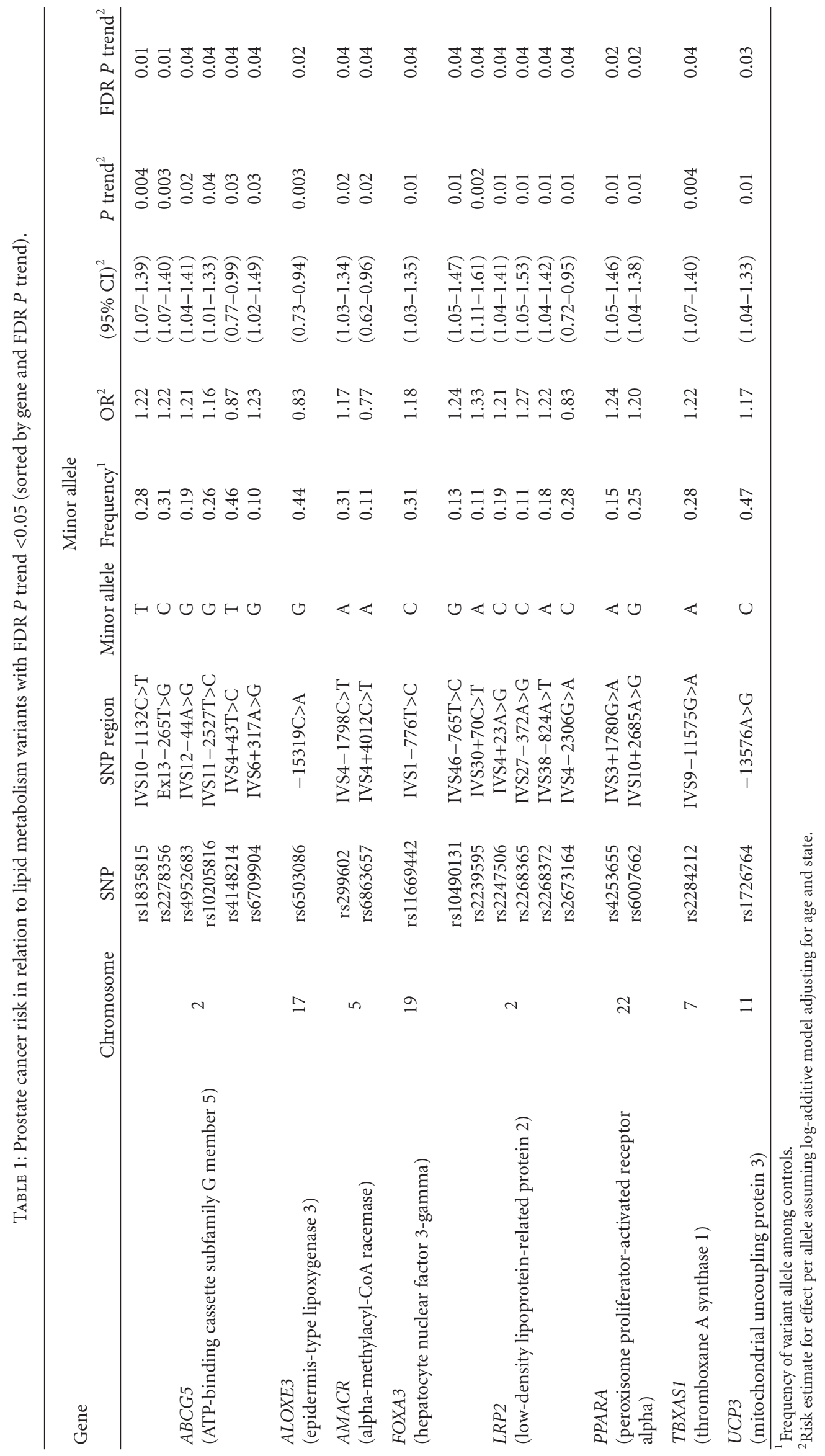




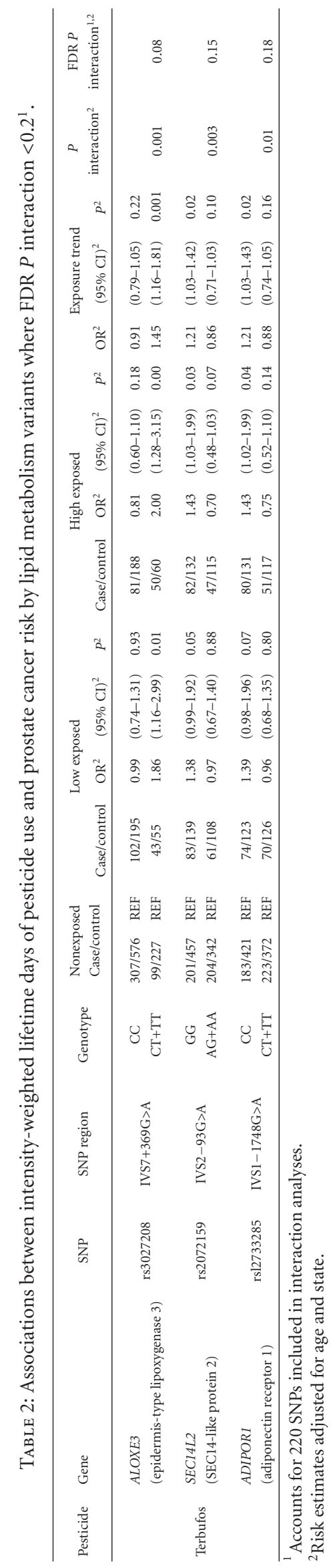




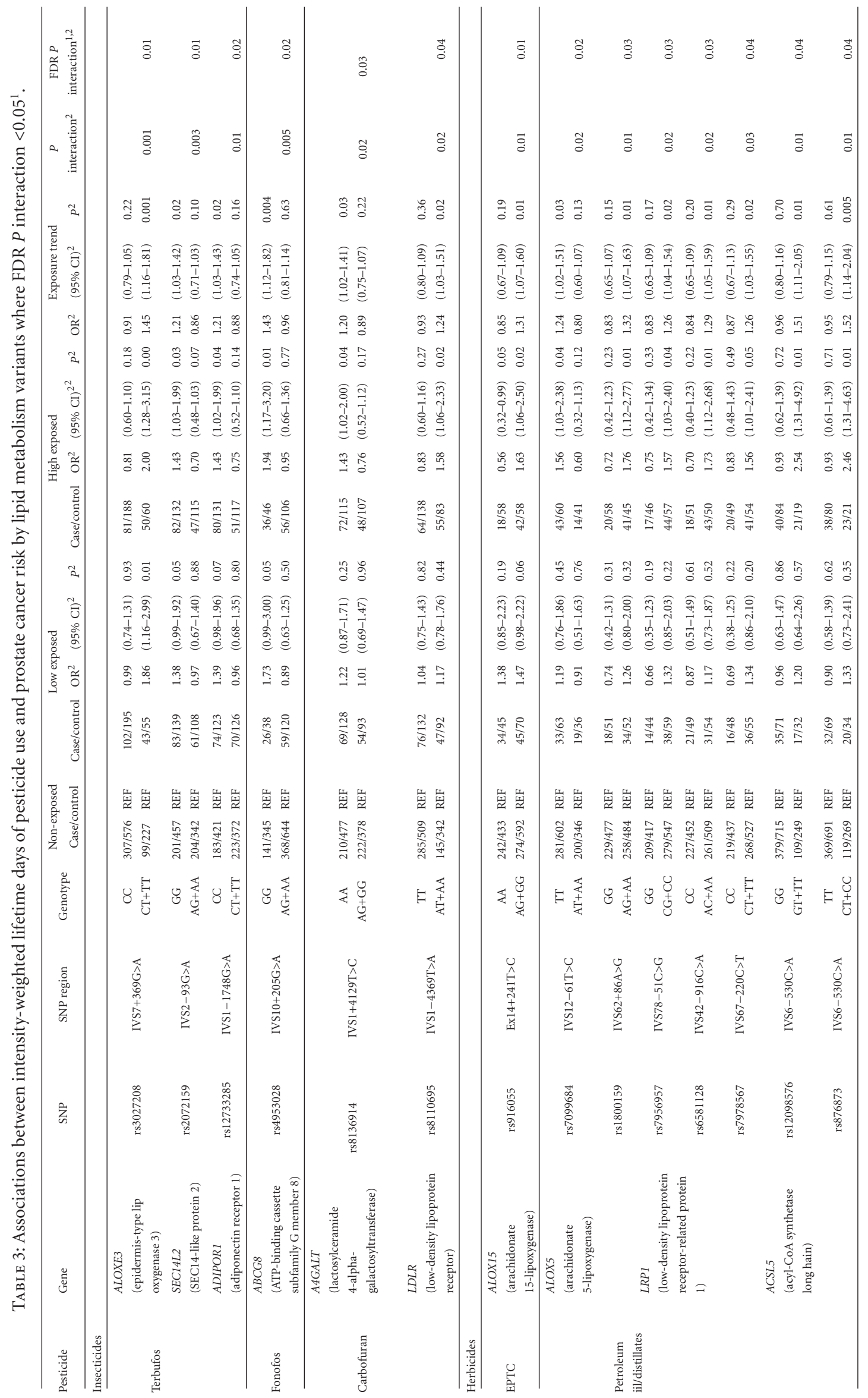




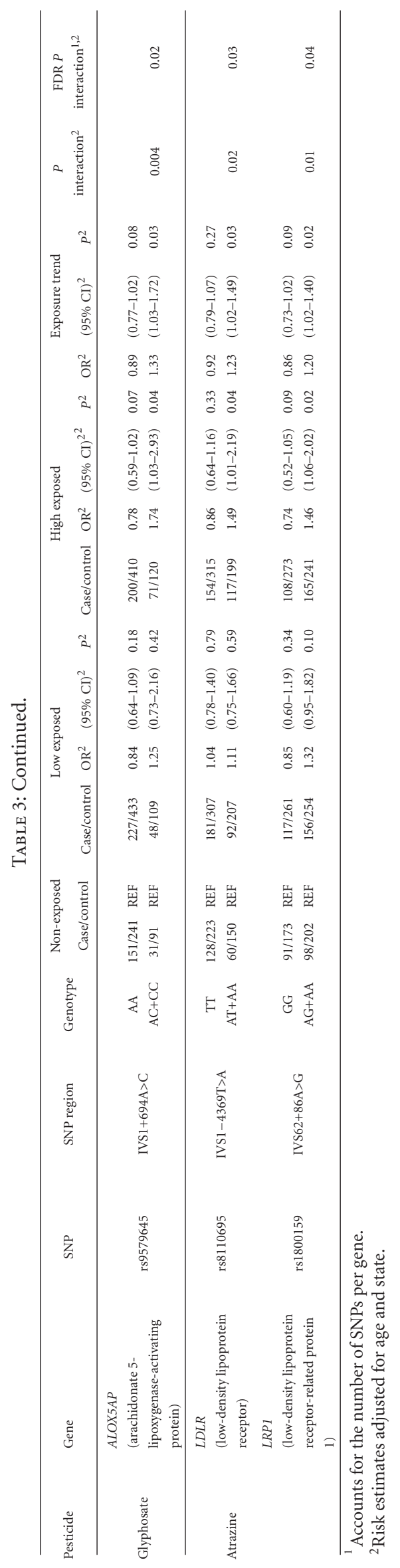


and $L D L R$ rs8110695 (FDR $P$ interaction=0.03) as well as LRP1 rs1800159 (FDR $P$-interaction $=0.04$ ), which, as mentioned above, also interacted with petroleum oil.

\section{Discussion}

In this nested case-control study, we evaluated the interaction between pesticide use and SNPs in genes related to lipid metabolism. We found 17 interactions that were robust to multiple comparison adjustment and displayed a significant monotonic increase in prostate cancer risk with increasing pesticide use in one genotype group and no significant association in the other genotype group. With these criteria, our analyses yielded seven pesticides (atrazine, carbofuran, EPTC, fonofos, glyphosate, petroleum oil/distillate, and terbufos) and 15 SNPs in 11 genes (ABCG8, ACSL5, ADIPOR1, ALOX5, ALOX5AP, ALOX15, ALOXE3, A4GALT, LDLR, LRP1, SEC14L2) of primary interest.

Increased prostate cancer risk has been associated with the insecticides (terbufos, fonofos, carbofuran) that we found to interact with lipid metabolism genes in previous studies in the AHS. Terbufos was suggestively linked to prostate cancer [32], but significantly with aggressive prostate cancer [4], and showed evidence of interaction with variants in the 8q24 region [9] and xenobiotic metabolism genes [10]. Fonofos has been linked to prostate cancer $[3,6]$ and aggressive prostate cancer [4], and interacted significantly with variants in the $8 \mathrm{q} 24$ region, and the nucleotide and base excision repair genes $[11,12]$. Similarly, carbofuran has been linked to prostate cancer [3], and interacted with variants in the nucleotide excision repair pathway [12]. In contrast to the insecticides, none of the four herbicides we identified have been associated with prostate cancer risk in main effect studies in the AHS (petroleum oil $[3,4]$, EPTC [3, 4, 33], atrazine [34, 35], glyphosate [36]). However, in previous analyses in the prostate nested case-control study, petroleum oil interacted with xenobiotic metabolism genes [10].

Our findings support the hypothesis that genetic variation in lipid metabolism genes might modify the associations between pesticides and prostate cancer; however, the biologic mechanisms are unclear. Organochlorines are lipophilic and accumulate in animal and human adipose tissue $[37,38]$; however, none of the six organochlorines we evaluated interacted significantly with the SNPs we examined. This may be due to organochlorines being of the market for approximately 30 years and having a very small number of participants who were exposed to these pesticides. Some pesticides in other classes have lipophilic properties or are metabolized via lipid-related mechanisms $[37,38]$. For example, organosphosphates have been shown to inhibit triglycerides and lipoprotein lipases in laboratory mice $[22,39,40]$. Two (terbufos and fonofos) of the nine organophophates we examined interacted significantly with lipid metabolism gene variants and were associated with an increased risk of prostate cancer. Terbufos interacted with a variant in ALOXE3 (arachidonate lipoxygenase 3), which is involved in a lipoxygenase reaction of a fatty acid that is converted to a molecule involved in the proliferation and specialization of the lipid membrane of the epidermis [41]. Terbufos also interacted with ADIPOR1 (adiponectin receptor 1) that encodes the adiponectin receptor and mediates ligand activities, fatty acid oxidation, and glucose uptake by adiponectin [42]; and SEC14L2 (SEC14-like protein 2) that is involved in lipid-binding and cholesterol biosynthesis [43]. Fonofos interacted with a variant in ABCG8 (ATP-binding cassette, sub-family G member 8), which encodes a protein that is a member of the ATPbinding cassette transporters that promotes biliary excretion of sterols [44]. Carbofuran belongs to another class of insecticide, carbamates, that inhibits cholinesterase and has been shown to generate reactive oxygen species and induce lipid peroxidation [45]. Carbofuran interacted with A4GALT (alpha 1,4-galactosyltransferase) which is involved in the formation of a glycolipid that provides cellular energy [46].

Among the herbicides, EPTC, classified as a thiocarbamate, has been reported to disrupt the biosynthesis of lipid metabolism in plants [47]. EPTC interacted with ALOX15, another gene in the lipoxygenase family that is involved in the metabolism of arachidonic and linoleic acid. Petroleum oil, which contains a mixture of hydrocarbons and is also lipophilic, is unique in that it is used as a herbicide, but also as an additive to other pesticides, and so it may have a wide variability in use [48]. It interacted with variants in three genes: ALOX5 (arachidonate 5-lipoxygenase), which transforms essential fatty acids into leukotrienes [49]; LRP1 (low-density lipoprotein receptor related protein 1), which regulates a large endocytic receptor that functions in lipoprotein transport [50]; ACSL5 (acyl-CoA synthetase long-chain 5) which converts free long-chain fatty acid to fatty acyl-CoA esters, and thereby plays a key role in lipid biosynthesis and fatty acid degradation [51]. Atrazine, a triazine herbicide, reported to be an endocrine disruptor in animals, was also shown to affect the lipid profiles of liver and muscle in fish [52]. It interacted with two genes belonging to the lowdensity lipoprotein receptor family (LDLR and LRP1). The low-density lipoprotein receptor (LDLR) is involved in the regulation of cholesterol homeostasis by receptor-mediated endocytosis [53].

While there is biologic plausibility to the interactions we observed, the specific biological mechanisms underlying these interactions are unclear. None of the SNPs we identified in our analysis are known to be functional or have been identified in published GWAS findings for prostate cancer (http://www.genome.gov/gwastudies/) [54]; however, they may be in linkage disequilibrium with other functional variants. Research conducted on the role of lipid metabolism in prostate carcinogenesis suggests that the mechanisms are complex, involving an array of intermediate compounds that can be modified by other pathways as well as various genetic, anthropometric, dietary, and lifestyle factors [55]. It is also possible that mechanisms other than lipid metabolism might play a role in the interactions we observed since most of the genes we identified have multiple functions. For example, genes within the arachidonic acid family play an important role in immunity and inflammation [56]. Furthermore, considering the findings from previous analyses from the nested case-control study of prostate cancer within AHS, we 
are seeing some of the same pesticides, such as terbufos, fonofos, carbofuran, EPTC, and petroleum oil, interacting with SNPs in multiple genetic pathways, suggesting that the relationship between pesticides and prostate cancer may involve multiple biologic processes.

In this analysis, we examined SNPs with a minor allele frequency (MAF) $>10 \%$ because of limited power below this MAF threshold and restricted analyses to SNPs with a main effect association with prostate cancer at $P<0.2$ to focus on SNPs potentially more associated with prostate cancer. However, by doing this, we may have excluded important SNPs that modify risk. Interaction analyses were conducted using the dominant genetic model since the number of participants in the homozygous variant group was often small; however, this may have resulted in loss of statistical power if another genetic model was more appropriate. Although some of our findings may be false-positives, we adopted a two-stage interaction approach and utilized FDR to limit this possibility. In addition, we focused our interpretations on interactions with positive monotonic associations between pesticide use and prostate cancer in one genotype and no significant association in the other genotype, which have greater biological plausibility than qualitative interactions, but could have resulted in exclusions of potentially relevant interactions. Due to small sample size we were unable to evaluate interactions by prostate cancer stage or grade.

In conclusion, we observed several positive interactions between pesticide use and variants in lipid metabolism genes. These findings suggest that lipid metabolism may be involved in the associations between pesticides and prostate cancer; however, little is known about the biologic mechanisms underlying these interactions. Furthermore, since this is the first study to explore the interaction between specific pesticide use and variants of genes in the lipid metabolism pathway, our results require replication.

\section{Acknowledgments}

This paper was supported by the Intramural Research Program of the NCI, Division of Cancer Epidemiology and Genetics (Z01CP010119) and NIEHS (Z01ES049030), NIH. None of the authors has any actual or potential competing financial interests.

\section{References}

[1] G. Van Maele-Fabry and J. L. Willems, "Prostate cancer among pesticide applicators: a meta-analysis," International Archives of Occupational and Environmental Health, vol. 77, no. 8, pp. 559-570, 2004.

[2] G. Van Maele-Fabry, V. Libotte, J. Willems, and D. Lison, "Review and meta-analysis of risk estimates for prostate cancer in pesticide manufacturing workers," Cancer Causes and Control, vol. 17, no. 4, pp. 353-373, 2006.

[3] M. C. R. Alavanja, C. Samanic, M. Dosemeci et al., "Use of agricultural pesticides and prostate cancer risk in the agricultural health study cohort," American Journal of Epidemiology, vol. 157, no. 9, pp. 800-814, 2003.

[4] S. Koutros, M. C. R. Alavanja, J. H. Lubin et al., "An update of cancer incidence in the agricultural health study," Journal of
Occupational and Environmental Medicine, vol. 52, no. 11, pp. 1098-1105, 2010.

[5] R. Mahajan, M. R. Bonner, J. A. Hoppin, and M. C. R. Alavanja, "Phorate exposure and incidence of cancer in the agricultural health study," Environmental Health Perspectives, vol. 114, no. 8, pp. 1205-1209, 2006.

[6] R. Mahajan, A. Blair, C. F. Lynch et al., "Fonofos exposure and cancer incidence in the Agricultural Health Study," Environmental Health Perspectives, vol. 114, no. 12, pp. 18381842, 2006.

[7] S. M. Lynch, R. Mahajan, L. E. Beane Freeman, J. A. Hoppin, and M. C. R. Alavanja, "Cancer incidence among pesticide applicators exposed to butylate in the Agricultural Health Study (AHS)," Environmental Research, vol. 109, no. 7, pp. 860-868, 2009.

[8] C. H. Christensen, E. A. Platz, G. Andreotti et al., "Coumaphos exposure and incident cancer among male participants in the Agricultural Health Study (AHS)," Environmental Health Perspectives, vol. 118, no. 1, pp. 92-96, 2010.

[9] S. Koutros, L. E. Beane Freeman, S. I. Berndt et al., "Pesticide use modifies the association between genetic variants on chromosome 8q24 and prostate cancer," Cancer Research, vol. 70, no. 22, pp. 9224-9233, 2010.

[10] S. Koutros, G. Andreotti, S. I. Berndt et al., "Xenobioticmetabolizing gene variants, pesticide use, and the risk of prostate cancer," Pharmacogenetics and Genomics, vol. 21, no. 10, pp. 615-623, 2011.

[11] K. H. Barry, S. Koutros, S. I. Berndt et al., "Genetic variation in base excision repair pathway genes, pesticide exposure, and prostate cancer risk," Environmental Health Perspectives, vol. 119, no. 12, pp. 1726-1732, 2011.

[12] K. H. Barry, S. Koutros, G. Andreotti et al., "Genetic variation in nucleotide excision repair pathway genes, pesticide exposure and prostate cancer risk," Carcinogenesis, vol. 33, no. 2, pp. 331-337, 2012.

[13] D. I. Feig, L. C. Sowers, and L. A. Loeb, "Reverse chemical mutagenesis: Identification of the mutagenic lesions resulting from reactive oxygen species-mediated damage to DNA," Proceedings of the National Academy of Sciences of the United States of America, vol. 91, no. 14, pp. 6609-6613, 1994.

[14] L. Wuermli, M. Joerger, S. Henz et al., "Hypertriglyceridemia as a possible risk factor for prostate cancer," Prostate Cancer and Prostatic Diseases, vol. 8, no. 4, pp. 316-320, 2005.

[15] J. V. Swinnen, T. Roskams, S. Joniau et al., "Overexpression of fatty acid synthase is an early and common event in the development of prostate cancer," International Journal of Cancer, vol. 98, no. 1, pp. 19-22, 2002.

[16] J. V. Swinnen, H. Heemers, T. Van De Sande et al., "Androgens, lipogenesis and prostate cancer," Journal of Steroid Biochemistry and Molecular Biology, vol. 92, no. 4, pp. 273-279, 2004.

[17] R. W. Mahley and S. C. Rall, "Apolipoprotein E: far more than a lipid transport protein," Annual Review of Genomics and Human Genetics, vol. 1, no. 2000, pp. 507-537, 2000.

[18] H. Knoblauch, A. Bauerfeind, M. R. Toliat et al., "Haplotypes and SNPs in 13 lipid-relevant genes explain most of the genetic variance in high-density lipoprotein and low-density lipoprotein cholesterol," Human Molecular Genetics, vol. 13, no. 10, pp. 993-1004, 2004.

[19] S. Lehrer, "Possible relationship of the apolipoprotein E (ApoE) $\varepsilon 4$ allele to prostate cancer," British Journal of Cancer, vol. 78, no. 10, p. 1398, 1998.

[20] S. L. Zheng, B. L. Chang, D. A. Faith et al., "Sequence variants of $\alpha$-methylacyl-CoA racemase are associated with prostate 
cancer risk," Cancer Research, vol. 62, no. 22, pp. 6485-6488, 2002.

[21] S. E. Daugherty, Y. Y. Shugart, E. A. Platz et al., "Polymorphic variants in $\alpha$-methylacyl-CoA racemase and prostate cancer," Prostate, vol. 67, no. 14, pp. 1487-1497, 2007.

[22] F. Matsumura, "Mechanism of action of dioxin-type chemicals, pesticides, and other xenobiotics affecting nutritional indexes," American Journal of Clinical Nutrition, vol. 61, no. 3, pp. 695S-701S, 1995.

[23] G. B. Quistad, S. N. Liang, K. J. Fisher, D. K. Nomura, and J. E. Casida, "Each lipase has a unique sensitivity profile for organophosphorus inhibitors," Toxicological Sciences, vol. 91, no. 1, pp. 166-172, 2006.

[24] M. C. R. Alavanja, D. P. Sandler, S. B. McMaster et al., "The agricultural health study," Environmental Health Perspectives, vol. 104, no. 4, pp. 362-369, 1996.

[25] J. Coble, K. W. Thomas, C. J. Hines et al., "An updated algorithm for estimation of pesticide exposure intensity in the agricultural health study," International Journal of Environmental Research and Public Health, vol. 8, no. 12, pp. 46084622, 2011.

[26] C. S. Carlson, M. A. Eberle, M. J. Rieder, Q. Yi, L. Kruglyak, and D. A. Nickerson, "Selecting a maximally informative set of single-nucleotide polymorphisms for association analyses using linkage disequilibrium," American Journal of Human Genetics, vol. 74, no. 1, pp. 106-120, 2004.

[27] S. Purcell, B. Neale, K. Todd-Brown et al., "PLINK: a tool set for whole-genome association and population-based linkage analyses," American Journal of Human Genetics, vol. 81, no. 3, pp. 559-575, 2007.

[28] J. C. Barrett, B. Fry, J. Maller, and M. J. Daly, "Haploview: analysis and visualization of LD and haplotype maps," Bioinformatics, vol. 21, no. 2, pp. 263-265, 2005.

[29] C. E. Murcray, J. P. Lewinger, and W. J. Gauderman, "Geneenvironment interaction in genome-wide association studies," American Journal of Epidemiology, vol. 169, no. 2, pp. 219-226, 2009.

[30] Y. Benjamini and Y. Hochberg, "Controlling the false discovery rate: a practical and powerful approach to multiple testing," Journal of the Royal Statistical Society B, vol. 57, no. 1, pp. 289300, 1995.

[31] N. S. Weiss, "Subgroup-specific associations in the face of overall null results: should we rush in or fear to tread?" Cancer Epidemiology Biomarkers and Prevention, vol. 17, no. 6, pp. 1297-1299, 2008.

[32] M. R. Bonner, B. A. Williams, J. A. Rusiecki et al., "Occupational exposure to terbufos and the incidence of cancer in the agricultural health study," Cancer Causes and Control, vol. 21, no. 6, pp. 871-877, 2010.

[33] D. M. van Bemmel, K. Visvanathan, L. E. Beane Freeman, J. Coble, J. A. Hoppin, and M. C. R. Alavanja, "S-ethyl-N,Ndipropylthiocarbamate exposure and cancer incidence among male pesticide applicators in the agricultural health study: a prospective cohort," Environmental Health Perspectives, vol. 116, no. 11, pp. 1541-1546, 2008.

[34] J. A. Rusiecki, A. De Roos, W. J. Lee et al., "Cancer incidence among pesticide applicators exposed to Atrazine in the agricultural health study," Journal of the National Cancer Institute, vol. 96, no. 18, pp. 1375-1382, 2004.

[35] L. E. Beane Freeman, J. A. Rusiecki, J. A. Hoppin et al., "Atrazine and cancer incidence among pesticide applicators in the Agricultural Health Study (1994-2007)," Environmental Health Perspectives, vol. 119, no. 9, pp. 1253-1259, 2011.
[36] A. J. De Roos, A. Blair, J. A. Rusiecki et al., "Cancer incidence among glyphosate-exposed pesticide applicators in the Agricultural Health Study," Environmental Health Perspectives, vol. 113, no. 1, pp. 49-54, 2005.

[37] International Agency for Research on Cancer, "Occupational exposures in insecticide application, and some pesticides," IARC Monographs on the Evaluation of Carcinogenic Risks to Humans, vol. 53, pp. 5-586, 1991.

[38] A. M. Soto, C. Sonnenschein, K. L. Chung, M. F. Fernandez, N. Olea, and F. Olea Serrano, "The E-SCREEN assay as a tool to identify estrogens: an update on estrogenic environmental pollutants," Environmental Health Perspectives, vol. 103, no. 7, pp. 113-122, 1995.

[39] H. D. Peters, P. Selhorst, V. Dinmendahl, K. U. Helm, and P. S. Schönhöfer, "Effect of paraoxon and antidotes on lipolysis of isolated fat cells," Archives of Toxicology, vol. 30, no. 2, pp. 139-145, 1973.

[40] J. P. Buchet, H. Roels, and R. Lauwerys, "Further characterization of mono and diglyceride lipases in rat tissues," Life Sciences, vol. 14, no. 2, pp. 371-385, 1974.

[41] J. W. Baynes and M. G. Dominiczak, Medical Biochemistry, Elsvier Mosby, 2nd edition, 2005.

[42] T. Yamauchi, J. Kamon, Y. Ito et al., "Cloning of adiponectin receptors that mediate antidiabetic metabolic effects," Nature, vol. 423, no. 6941, pp. 762-769, 2003.

[43] I. Dunham, N. Shimizu, B. A. Roe et al., "The DNA sequence of human chromosome 22," Nature, vol. 402, no. 6761, pp. 489-495, 1999.

[44] G. Schmitz, T. Langmann, and S. Heimerl, "Role of ABCG1 and other ABCG family members in lipid metabolism," Journal of Lipid Research, vol. 42, no. 10, pp. 1513-1520, 2001.

[45] A. Kamboj, R. Kiran, and R. Sandhir, "N-Acetylcysteine ameliorates carbofuraninduced alterations in lipid composition and activity of membrane bound enzymes," Molecular and Cellular Biochemistry, vol. 286, no. 1-2, pp. 107-114, 2006.

[46] J. J. Keusch, S. M. Manzella, K. A. Nyame, R. D. Cummings, and J. U. Baenziger, "Cloning of GB3 synthase, the key enzyme in globo-series glycosphingolipid synthesis, predicts a family of $\alpha 1,4$-glycosyltransferases conserved in plants, insects, and mammals," Journal of Biological Chemistry, vol. 275, no. 33, pp. 25315-25321, 2000.

[47] ICI Americas Inc., Materials Safety Data Sheet for Eptam Technical, ICI, 1992.

[48] F. M. Ashton and T. J. Monaco, Weed Science, John Wiley and Sons, New York, NY, USA, 3rd edition, 1991.

[49] J. H. Dwyer, H. Allayee, K. M. Dwyer et al., "Arachidonate 5-lipoxygenase promoter genotype, dietary arachidonic acid, and atherosclerosis," New England Journal of Medicine, vol. 350, no. 1, pp. 29-37, 2004.

[50] J. Herz and D. Y. Hui, "Lipoprotein receptors in the vascular wall," Current Opinion in Lipidology, vol. 15, no. 2, pp. 175181, 2004.

[51] E. Soupene and F. A. Kuypers, "Mammalian long-chain acylCoA synthetases," Experimental Biology and Medicine, vol. 233, no. 5, pp. 507-521, 2008.

[52] P. A. MacLennan, E. Delzell, N. Sathiakumar et al., "Cancer incidence among triazine herbicide manufacturing workers," Journal of Occupational and Environmental Medicine, vol. 44, no. 11, pp. 1048-1058, 2002.

[53] J. L. Goldstein, R. A. DeBose-Boyd, and M. S. Brown, "Protein sensors for membrane sterols," Cell, vol. 124, no. 1, pp. 35-46, 2006. 
[54] L.A. Hindorff, J. MacArthur, A. Wise et al., "A Catalog of Published Genome-Wide Association Studies," http://www .genome.gov/gwastudies/.

[55] E. A. Platz and E. Giovannucci, Prostate Cancer in Cancer Epidemiology and Prevention, D. Schottenfeld and J. F. Fraumeni, Eds., Oxford University Press, Oxford, UK, 3rd edition, 2006.

[56] M. Hughes-Fulford, C. F. Li, J. Boonyaratanakornkit, and S. Sayyah, "Arachidonic acid activates phosphatidylinositol 3-kinase signaling and induces gene expression in prostate cancer," Cancer Research, vol. 66, no. 3, pp. 1427-1433, 2006. 


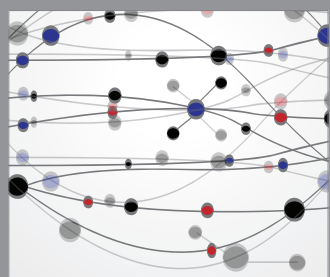

The Scientific World Journal
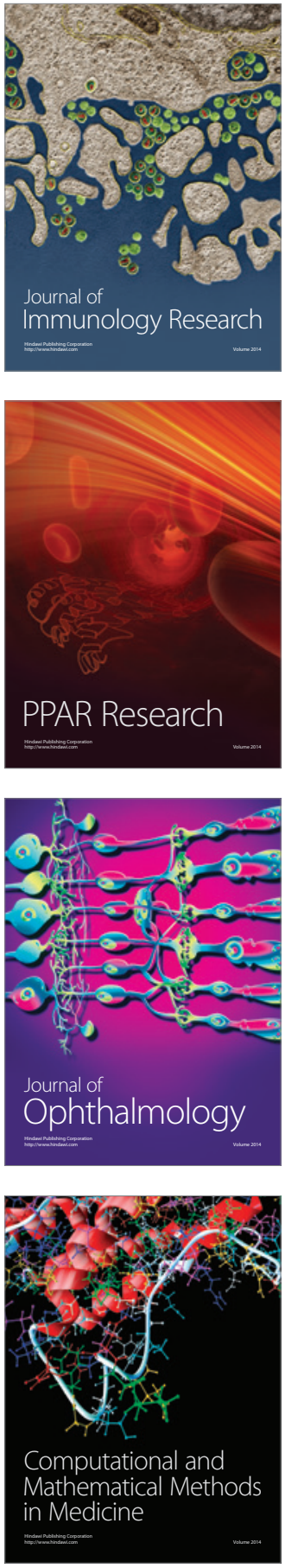

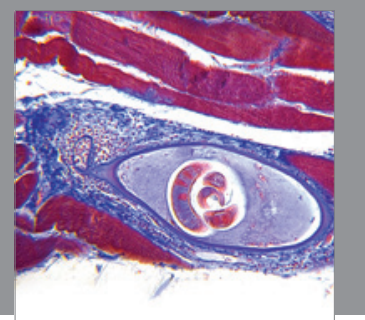

Gastroenterology

Research and Practice
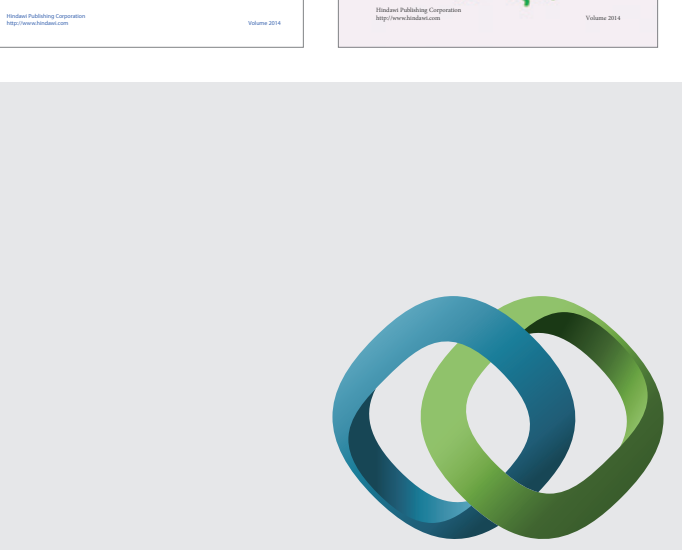

\section{Hindawi}

Submit your manuscripts at

http://www.hindawi.com
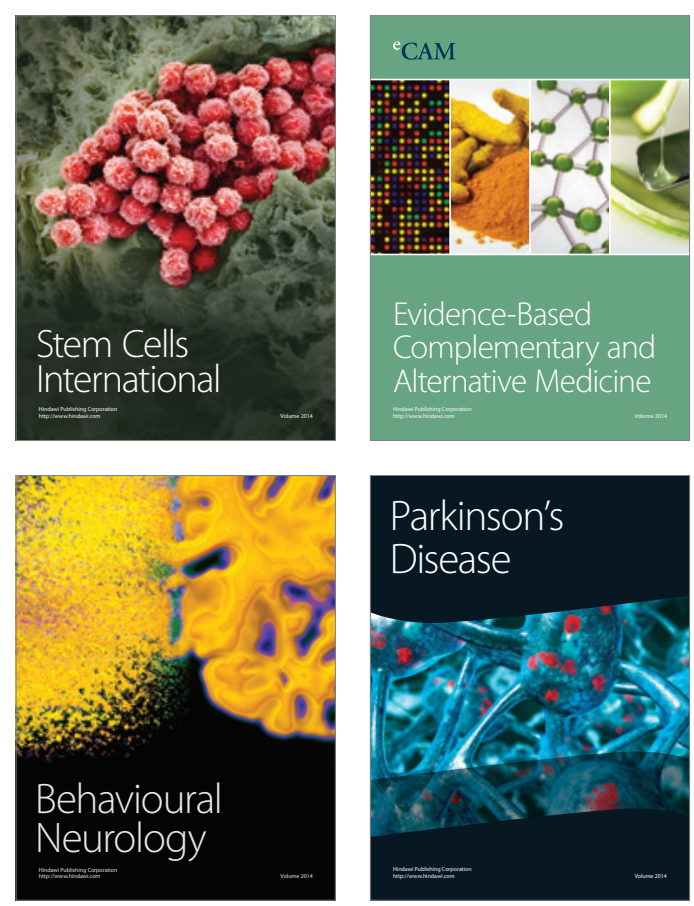

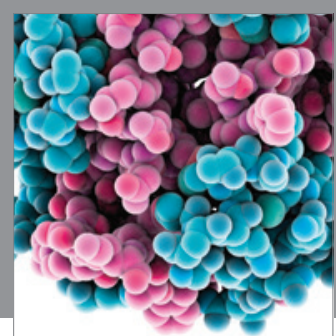

Journal of
Diabetes Research

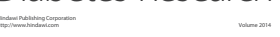

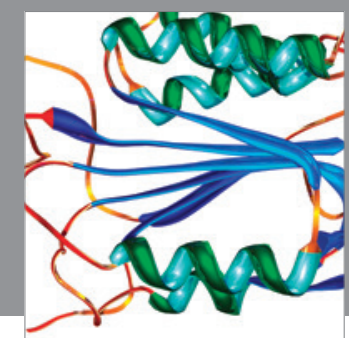

Disease Markers
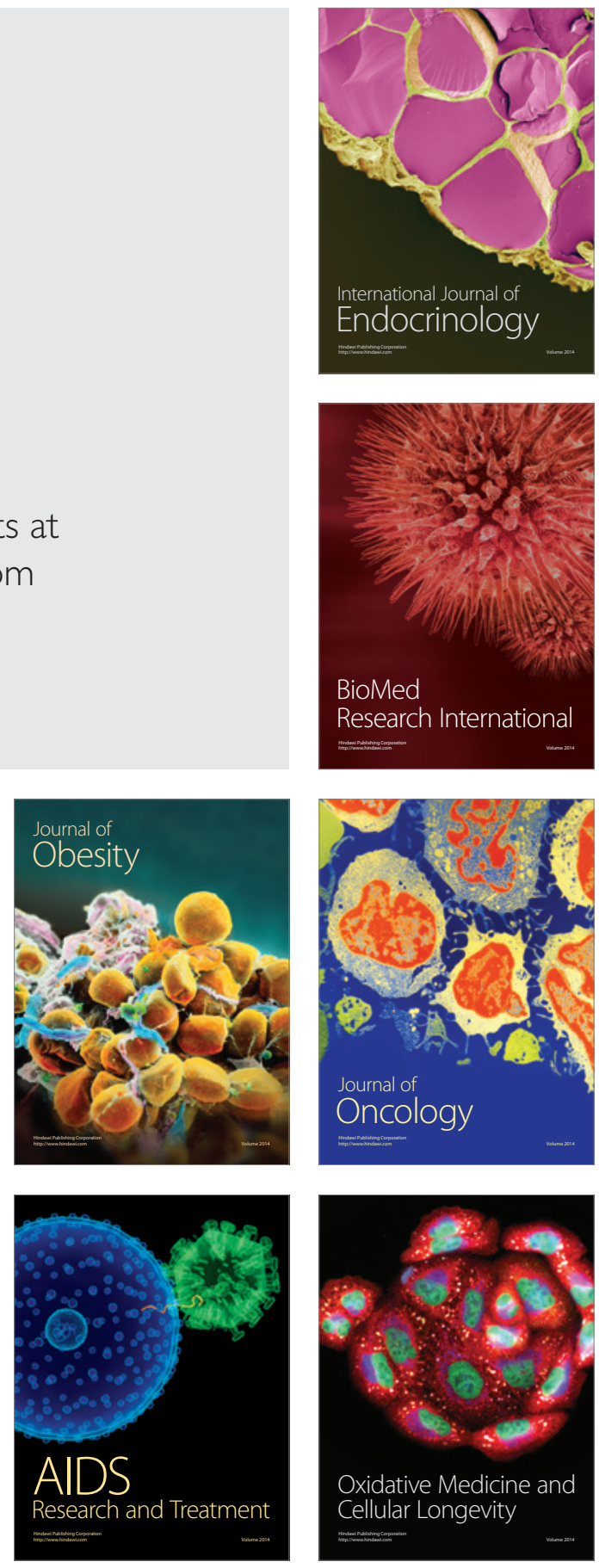